\title{
Tillfälligt inflöde av magra och aggressiva talgoxar Parus major under höstflyttningen på Utklippan
}

\author{
Occasional influx of lean and aggressive Great Tits Parus major during autumn \\ migration at Utklippan
}

ROLF LARSSON

\begin{abstract}
On October 13th and 14th, 2014 there was an influx of Great Tits Parus major to the island of Utklippan, $15 \mathrm{~km}$ off the coast of south-eastern Sweden. During these two days, 158 Great Tits were trapped, ringed and weighed. Most of them were very light; $90.4 \%$ of the males and $96.5 \%$ of the females had a body mass below the estimated lean body mass (males $=17.3 \mathrm{~g}$, females $=16.3$ $\mathrm{g}$ ). The mean body mass during the influx days was $1.5 \mathrm{~g}$ (males) and $1.56 \mathrm{~g}$ (females) below these lean body mass values. The birds had probably left the eastern coast of the Baltic Sea the day before and then met unfavourable

weather with rain and strong head-winds. Probably they had been disoriented and forced to fly for at least 8 hours or even up to 20 hours over open sea before reaching Utklippan. Many of the Great Tits were very aggressive and they attacked other species and killed them by pecking at the head to eat the brain of the victims.

Rolf Larsson, Utklippan Bird Observatory, Flyetvägen 27, SE 37300 Jämjö, Sweden

Email: rolflar@gmail.com
\end{abstract}

Received 7 April 2015, Accepted 16 September 2015, Editor: D. Hasselquist

\section{Inledning}

Utklippan ligger i öppna havet $15 \mathrm{~km}$ sydsydväst Torhamns udde på fastlandet i sydöstra Blekinge och består i huvudsak av två större öar (totalt 13 ha) som i stort sett saknar träd. Vid Utklippans fågelstation har sammantaget 400000 fåglar ringmärkts sedan 1964 och fångst sker främst med slöjnät. Under gynnsamma väder- och vindförhållanden är näten uppe för fångst under dygnets ljusa timmar. Stationen är sedan 1972 bemannad av ideell personal vår och höst, totalt omkring 110 dagar per år.

Talgoxen är stannfågel i en stor del av de södra och centrala delarna av sitt utbredningsområde i Västpalearktis. Men invasionsrörelser inträffar oregelbundet i de norra delarna och arten kan ibland uppträda i stora antal. Många av de talgoxar som häckar i de nordvästra delarna av Ryssland flyttar, i huvudsak till Östersjöområdet, men ofta ännu längre (Cramp 1993). Talgoxen är i första hand en dagsträckare (Newton 2008).

Talgoxens sträckriktning är sydvästlig och medelhastigheten för höstflyttningen är 47,5 km per dygn och den högsta hastigheten som registrerats i Sverige för en talgoxe är $98 \mathrm{~km}$ per dygn (Fransson \& Hall-Karlsson 2008). Denna typ av ring- märkningsdata baserat på återfynd ger ett mått på flyttningshastighet, dvs. förflyttad sträcka över en tidsperiod på minst ett (men oftast många) dygn och inkluderar både flygnings- och rastningstid.

Studier av återfynd av talgoxar som ringmärkts i Baltikum och Polen visar att talgoxar som sträcker över land förflyttar sig i genomsnitt 33,2 km per dag (Nowakowski 2001). Maximala flyttningshastigheter var $231 \mathrm{~km}$ under samma dag och $353 \mathrm{~km}$ inom 24 timmar. Dock förflyttade sig $70 \%$ av talgoxarna bara mellan 18 och $44 \mathrm{~km}$ per dag. Ett fătal procent av de snabbaste flyttningsrörelserna ägde troligen rum vid kraftig medvind (Nowakowski 2001).

Under höstarna 1975 och 1976 förde sydostliga vindar talgoxar på bred front över Östersjön till den svenska kusten. Under dagar med den kraftigaste medvinden $(15 \mathrm{~m} / \mathrm{s}=54 \mathrm{~km} / \mathrm{h})$ hade talgoxarna en hastighet på hela $84 \mathrm{~km} / \mathrm{h}$ (Alerstam 1982). Men notera att detta avser radarstudier av fåglar som flyger över hav och är således ett mått på flygningshastighet, dvs. den hastighet en flygande fågel rör sig i relation till marken (inkluderar således effekter av med/motvind men inte rastningstid).

Dagsträckare såsom talgoxe börjar normalt sträcka strax före soluppgången, och sträcket är in- 
tensivast under de tidigaste morgontimmarna och försvagas framåt middagstid. Flera arter upphör då helt att sträcka. Men somliga arter uppvisar ibland även en sträcktopp på kvällen (Alerstam 1982).

Gemensamt för höst och vår är att flyttfåglarna sträcker intensivast när de har gynnsamma medvindar och att de undviker nederbördsområden. Vissa arter, som exempelvis finkar, är redo att
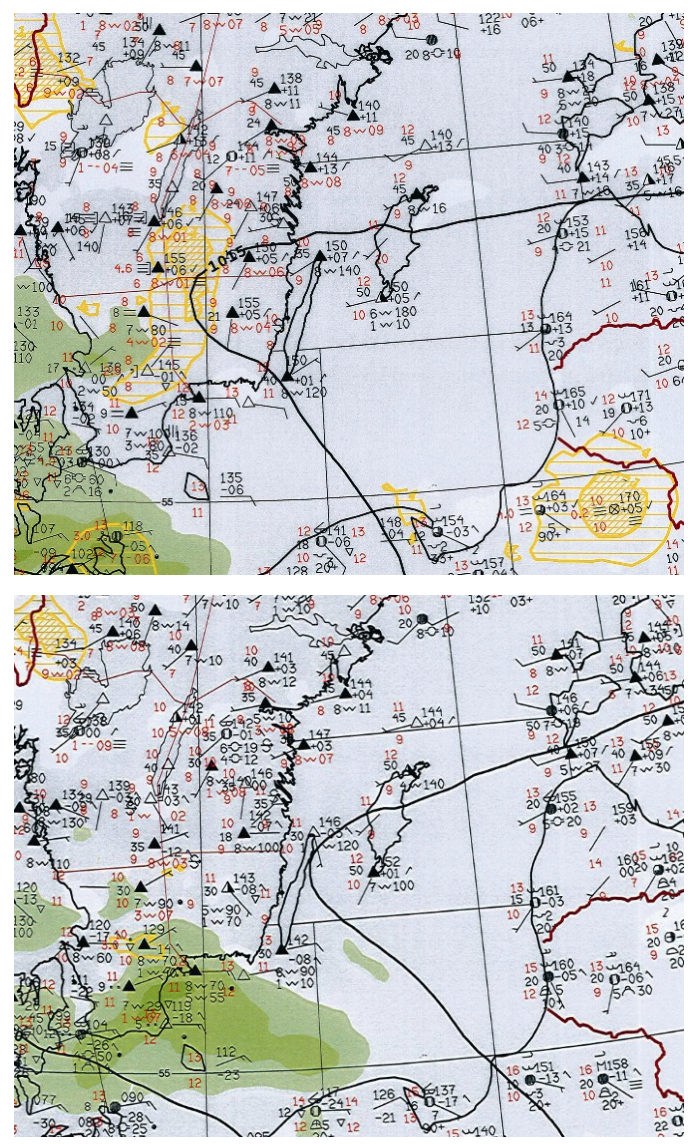

Figur 1. Vädersituationen den 13 oktober 2014 klockan 11 och 14 svensk sommartid. Ett regnområde (grönt och grått område på kartan) rör sig från sydväst in över södra Östersjön. Vid den baltiska kusten är vindarna svaga och sydvästliga. Över Kaliningradområdet finns på morgonen dis och dimma (gult). Symbolen med en punkt och ett långt streck anger vindriktningen (det blåser in mot punkten). Väderkartor från SMHI.

The weather situation on October $13^{\text {th }}$ at 11 and 14 CEST. An area of rain (green and grey areas on the map) moves from $S W$ over the southern part of the Baltic Sea. At the coast of the Baltic states, the winds are weak from $S W$. Over the Kaliningrad area there is mist and fog (yellow area on the map). The symbol with a dot and a long line indicates the wind direction (the wind blows along the line towards the dot). Weather maps from SMHI. sträcka även i motvind (Alerstam 1982). Oberoende av vädret vid startpunkten för sträcket, kan flyttande fåglar stöta på dåliga väderförhållanden under färden. Radarstudier har visat att fåglar, som brukar flytta över land men som råkar befinna sig över hav, vanligtvis blir desorienterade när de kommer in i moln eller dimbankar. De flyger runt $i$ alla riktningar och driver undan med vinden eller flyger aktivt med vinden för att nå klarare väder (Newton 2008).

\section{Väderläge}

I början av oktober 2014 präglades vädret på Utklippan av perioder med ostliga vindar med avbrott för några dagar med i stort sydvästliga vindar. Under senare delen av natten mellan den 12-13 oktober slog vinden om från nordväst till ost.

\section{3 oktober}

Vid den lettiska och estniska kusten var vindarna under den ljusa delen av den 13 oktober svaga $(<5$ $\mathrm{m} / \mathrm{s}$ ) och västliga. I Gdanskbukten var det på morgonen så gott som vindstilla men utmed kusten låg dis (sikt $<5 \mathrm{~km}$ ) och in över land dimma (sikt $<1$ $\mathrm{km}$ ). Innanför kusten i Litauen och Lettland låg ett område med nedsatt sikt (sikt $<5 \mathrm{~km}$ ). Diset lättade vid middagstid, men i inlandet innanför Kaliningrad låg dimman kvar vid middagstid (Figur 1).

Samtidigt trängde ett regnområde in västerifrån över sydvästra Östersjön. Regnet rörde sig i en ostnordostlig bana. Det nådde Utklippan vid 14-tiden (svensk sommartid CEST), södra Gotland vid 18-tiden och den baltiska kusten sent på kvällen (Figur 2).

Vid Östergarn på Gotland rådde svaga sydvästliga vindar $(<5 \mathrm{~m} / \mathrm{s})$ fram till sen eftermiddag då vinden vred mot sydost. Fram på kvällen skedde en vindkantring på sydöstra Östersjön till nordost och på mellersta Östersjön till ost-nordost och ökande vind.

På Utklippan var vinden ostlig under hela dagen och vindstyrkan ökade från ca 3 m/s till $13 \mathrm{~m} / \mathrm{s}$. Sent på kvällen vred den över mot nordost. Vid ca klockan 13:30 kom en front med regn.

\section{4 oktober}

Under natten till den 14 oktober ökade den nordostliga vinden vid Östergarn till över $14 \mathrm{~m} / \mathrm{s}$ i medelvind, på Ölands södra udde $12 \mathrm{~m} / \mathrm{s}$. och på Utklippan $14 \mathrm{~m} / \mathrm{s}$. På Utklippan låg den friska till hårda vinden på från nordnordost under resten av dagen. 


\section{Händelse}

Den 13-14 oktober skedde ett kraftigt inflöde av talgoxar till Utklippan i den rådande ostliga till nordostliga vinden. Under dessa dagar dominerade talgoxarna fångsten vid Utklippans fågelstation. Samtliga talgoxar var i dålig kondition och vägde långt under vad som är normalt för årstiden. Åtskilliga av de utmärglade fåglarna dog på ön medan andra var mycket aggressiva och anföll och dödade andra fåglar för att komma åt föda.

\section{Fångst}

Efter en natt med relativt svaga ostliga vindar inträffade den 12 oktober ett kraftigt inflöde av

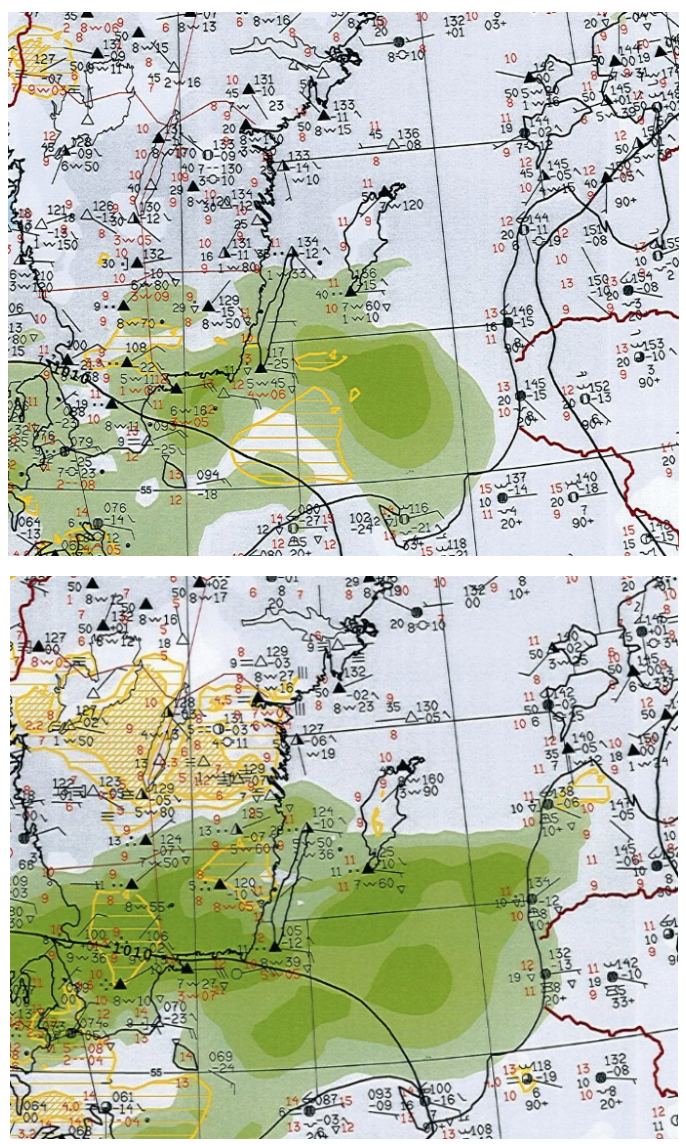

Figur 2. Vädersituationen den 13 oktober 2014 klockan 17 och 20 svensk sommartid. Symbolen med en punkt och ett långt streck anger vindriktningen (det blåser in mot punkten). Väderkartor från SMHI.

The weather situation on October $13^{\text {th }}$ at 17 and 20 CEST. The symbol with a dot and a long line indicates the wind direction (the wind blows along the line towards the dot). Weather maps from SMHI. kungsfågel (676 märka) och rödhake (162 märkta). Blott 11 talgoxar märktes den dagen.

Den 13 oktober minskade mängden kungsfågel och rödhake (176 respektive 52 märkta). Endast 8 talgoxar märktes. Klockan 14 drogs fångstnäten ihop på grund av ett passerande regnområde.

Redan på morgonen den 14 oktober började näten fyllas med talgoxar. På grund av att talgoxarna gick till hårt angrepp mot andra fåglar i näten måste nätfångsten avbrytas kl. 10:30. Då hade 158 talgoxar ringmärkts. Antalet kungsfåglar och rödhakar inskränkte sig till 6 respektive 19 märkta. Dagen därpå den 15 oktober kunde 24 talgoxar ringmärkas och en kontroll av en i Litauen ringmärkt talgoxe gjordes. Klockan 12 var det åter dags att dra ihop näten på grund av de aggressiva talgoxarna. Samma sak skedde klockan 11 den 16 oktober.

\section{Vikter}

Vid ringmärkningen den 14 oktober noterades att en stor del av talgoxarna var mycket magra och att de vägde klart mindre än normalt. För hanarna var medelvikten denna dag $15,80 \mathrm{~g}(\mathrm{n}=73$; Figur 3, Tabell 1). Detta ska jämföras med medelvikten för hanar under oktober 2007-2013 för Utklippan som låg på $16,99 \mathrm{~g}(\mathrm{U}$-test, $\mathrm{z}=7,82, \mathrm{p}<0,001)$, och för de närbelägna lokalerna Torhamns udde och Flyet (inlandslokal $2 \mathrm{~km}$ från kusten och $10 \mathrm{~km}$ nordnordost om Torhamns udde) var motsvarande värden 17,53 g respektive 18,54 g (Figur 2 och Tabell 1). För honorna på Utklippan var medelvikten den 14 oktober $14,74 \mathrm{~g}(\mathrm{n}=85)$. Detta är klart lägre än medelvikten för honor under oktober 2007-2013 på Utklippan $(15,95 \mathrm{~g}$; U-test, $\mathrm{z}=3,19, \mathrm{p}<0,001)$ och på de närbelägna lokalerna Torhamns udde $(16,43$ g) och Flyet (17,33 g; Figur 3 och Tabell 1).

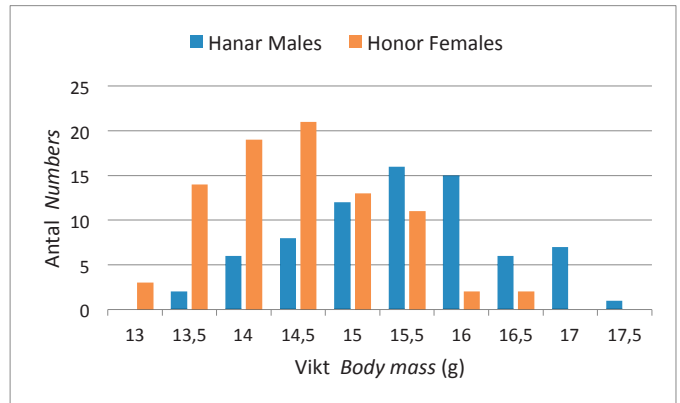

Figur 3. Viktfördelning hos de talgoxar som ringmärktes på Utklippan den 14 oktober 2014 fördelade på hanar och honor.

Body mass distribution of Great Tit males and females trapped and ringed at Utklippan on October 14th 2014. 
Tabell 1. Medelvikt för hanar och honor av talgoxe ringmärkta på Utklippan, Torhamn, Flyet och Ottenby. Mean body mass of male and female Great Tit ringed at Utklippan, Torhamn, Flyet and Ottenby.

\begin{tabular}{|c|c|c|c|c|c|}
\hline & & $\begin{array}{l}\text { Medelvikt (g) } \\
\text { Mean body mass }\end{array}$ & $\begin{array}{l}\text { Antal } \\
\text { Number }\end{array}$ & $\begin{array}{l}\text { Min-max } \\
(g)\end{array}$ & SD \\
\hline \multicolumn{6}{|c|}{ Hanar Males } \\
\hline Utklippan & 14 Okt 2014 & 15,80 & 73 & $13,9-17,7$ & 0,9 \\
\hline Utklippan & Okt 2007-2013 & 16,99 & 331 & $14,2-20,6$ & 1,14 \\
\hline Torhamn & Okt 2007-2013 & 17,53 & 138 & $14,0-20,6$ & 1,16 \\
\hline Flyet & Okt 2007-2013 & 18,54 & 449 & $15,2-21,6$ & 0,94 \\
\hline Ottenby & 14 Okt 2014 & 16,51 & 26 & $14,5-18,4$ & 0,89 \\
\hline \multicolumn{6}{|c|}{ Honor Females } \\
\hline Utklippan & 14 Okt 2014 & 14,74 & 85 & $13,3-16,7$ & 0,76 \\
\hline Utklippan & Okt 2007-2013 & 15,95 & 508 & $12,3-19,0$ & 1,03 \\
\hline Torhamn & Okt 2007-2013 & 16,43 & 269 & $13,7-19,1$ & 0,96 \\
\hline Flyet & Okt 2007-2013 & 17,33 & 452 & $14,8-20,8$ & 0,97 \\
\hline Ottenby & 14 Okt 2014 & 15,03 & 21 & $13,5-17,0$ & 0,83 \\
\hline
\end{tabular}

Vid Ottenby fågelstation fångades den 14 oktober 48 talgoxar och deras vikter var något högre än vad som noterades på Utklippan. Hanarnas medelvikt på $16,51 \mathrm{~g}(\mathrm{n}=26)$ var signifikant skild från medelvikten för de hanar som fångades på Utklippan samma dag (U-test, $\mathrm{z}=3,19, \mathrm{p}=0,001$ ). De honor som fångades på Ottenby den 14 oktober 2014 hade en medelvikt på $15,03 \mathrm{~g}(\mathrm{n}=21)$, vilket dock inte skiljde sig från medelvikten för de honor som fångades på Utklippan samma dag (U-test, $\mathrm{z}=1,46, \mathrm{p}=0,15)$.

Utklippan är en ren sträcklokal för talgoxar eftersom ön är isolerad och helt saknar häckande talgoxar. Också Torhamns udde måste betecknas som i huvudsak en sträcklokal, även om talgoxen häckar i närliggande skogsområden och i Torhamns samhälle. Flyet, som ligger nära kusten (ca $2 \mathrm{~km}$ inåt land och $10 \mathrm{~km}$ norr om Torhamn), är i viss mån en sträcklokal men där finns en lokal population av många häckande talgoxar. Medelvikten för både hanar och honor som fångades vid Torhamns udde och vid Flyet i oktober åren 2007-2013 var klart högre än medelvikten på Utklippan under samma period (Tabell 1). Vikterna skiljer sig också signifikant åt för båda könen mellan de tre platserna (Kruskal Wallis, $\mathrm{H}=315,44$, $\mathrm{df}=2, \mathrm{p}<0,001$ resp $\mathrm{H}=348,04, \mathrm{df}=2, \mathrm{p}<0,001)$.

\section{Aggressivt beteende}

I terrängen på Utklippan hittades talgoxar som dukat under och dött. Flera talgoxar var så utmattade att de dog i fångstnäten trots att näten hölls under ständig uppsikt.
Talgoxar sågs sitta på marken och äta på döda artfränder. Vid något tillfälle sågs också attacker mot artfränder som var så starkt försvagade att de inte förmådde försvara sig. Det förekom också ett flertal attacker mot fåglar av andra arter som fastnat i stationens fångstnät. Det var främst kungsfåglar, rödhakar, gärdsmygar, gransångare, järnsparvar och grönsiskor, som föll offer för talgoxarna. Attackerna började med att talgoxarna först hackade ut ögonen på offret för att sedan hacka sig in i hjärnan. De åt också av bröstmusklerna. Attacker mot talgoxar som fastnat i näten kunde inte konstateras. Troligen på grund av att de eventuella offren ännu förmådde värja sig.

\section{Tidigare inflöden av aggressiva talgoxar}

Inflöden med aggressiva talgoxar är inte vanligt förekommande. Jag känner bara till två liknande händelser från Utklippan. I slutet av oktober 2002 ringmärktes sammanlagt 259 talgoxar under fem dagar på Utklippan. Hanarna $(\mathrm{n}=131)$ hade en medelvikt på 17,01 g $(13,9-20,5 \mathrm{~g})$. Honornas $(\mathrm{n}=128)$ medelvikt var 15,78 g (13,1-18,5 g). De strömmade in med sydostliga och ostliga vindar. De gick till angrepp mot både artfränder och andra arter med följd att nätfångsten av fågel måste avbrytas flera dagar. Det andra inflödet inträffade under fyra dagar i slutet av oktober 2011. Vid det tillfället rådde sydostliga vindar. 195 talgoxar ringmärktes. Medelvikten för hanarna låg på 17,28 g (14,2-20,5 g; $\mathrm{n}=82)$ och för honorna 16,25 g $(13,4-19 \mathrm{~g} ; \mathrm{n}=$ 113). En talgoxe märkt i Rybachy, beläget vid Ku- 
riska lagunen i sydostligaste Östersjön) den 18 oktober kontrollerades den 27 oktober. Också dessa talgoxar gick till angrepp med följd att nätfångsten måste avbrytas under flera dagar. Medelvikterna vid dessa båda inflöden låg alltså väl över medelvikterna för den 14 oktober 2014. En förklaring till detta kan vara att talgoxarna 2002 och 2011 hade god medvind under passagen över Östersjön, medan detta inte var fallet 2014.

\section{Diskussion}

\section{Varifrån kom de magra talgoxarna?}

Massförekomsten av aggressiva talgoxar till Utklippan 13-14 oktober 2014 torde vara en del av ett inflöde av fåglar från östra sidan av Östersjön. Under 11-18 oktober ringmärktes många talgoxar på den västra sidan av Östersjön. Medan Svenska högarna i Stockholms skärgård hade ett tämligen konstant inflöde av talgoxar under hela denna period, var det först den 14 oktober och de följande dagarna som ett större antal talgoxar kom till Landsort i Stockholms skärgård, Ottenby på Öland och Utklippan. Falsterbo fick ett mindre inflöde först den 16 oktober (Figur 4).

Denna bild stöds av de kontroller av talgoxar som gjordes 14-18 oktober vid flera svenska fågelstationer (Svenska högarna, Ottenby, Utklippan och Falsterbo i sydvästra Skåne) av fåglar vilka tidigare ringmärkts i Finland (2), Litauen (1), Ryssland (1) och Polen (2). De båda finskmärkta fåglarna kontrollerades på Svenska högarna den 14 oktober, 3 respektive 6 dagar efter att de märkts vid Hangö i Finska viken. Dessa kontroller gjordes under de tidiga morgontimmarna (kl. 07-09). Den ryska fågeln ringmärktes vid Rybachy den 9 oktober och kontrollerades vid Ottenby den 14 oktober. Den ena av de polskmärkta talgoxarna, som kontrollerades vid Ottenby den 14 oktober, hade ringmärkts i oktober 2013 vid Krynica Morska i Gdanskbukten. Den andra som ringmärkts den 10 oktober 2014 vid Krynica Morska kontrollerades i Falsterbo 8 dagar senare. Den 15 oktober fångades på Utklippan en talgoxhona som ringmärkts vid Ventes Ragas vid Kuriska lagunen i Litauen. Märkningen hade dock skett den 9 mars 2014. Detta ger alltså inte någon information om varifrån fågeln lämnade den baltiska eller polska kusten under de aktuella dagarna, eller var den häckat under 2014.

För att flyga de ca 33 milen över Östersjön från den Baltiska kusten till Utklippan tar det med en teoretiskt beräknad hastighet på knappt $40 \mathrm{~km} / \mathrm{h}$ vid vindstilla förhållanden cirka 8 timmar (Alerstam 1982). Från den polska kusten norr om Gdansk skulle en flygtur på ca 20 mil, beräknat på samma sätt, ta 5 timmar. För talgoxar som i september 1975 flög över Östersjön till Ottenby, ca 30 mil, beräknades det ta drygt 5 timmar vid en medvind på ca $5 \mathrm{~m} / \mathrm{s}=18 \mathrm{~km} / \mathrm{h}$ och en registrerad medelhastighet på $57 \mathrm{~km} / \mathrm{h}$ (Lindholm 1978).

De talgoxar som fångades tidigt på morgonen den 14 oktober torde ha lämnat den baltiska kusten norr om Litauen någon gång på morgonen eller förmiddagen den 13:e trots att de inte hade medvind. Vindarna var västliga till sydvästliga men svaga. Det är inte troligt att de flög ut över Östersjön från Polen, Kaliningradområdet och södra Litauen eftersom det låg ett bälte med dis eller dimma över området från gryningen till fram på förmiddagen.

Talgoxarna hade alltså en svag motvind och därmed minskade deras flyghastighet. När de väl kommit ut över havet möttes de av den molnskärm som föregick det från sydväst annalkande regnområdet. Därmed kan fåglarnas möjlighet att

Figur 4. Antal ringmärkta talgoxar vid svenska fågelstationer under perioden 11-18 oktober 2014.

Number of Great Tits ringed at Swedish bird ringing stations during 11-18 October 2014

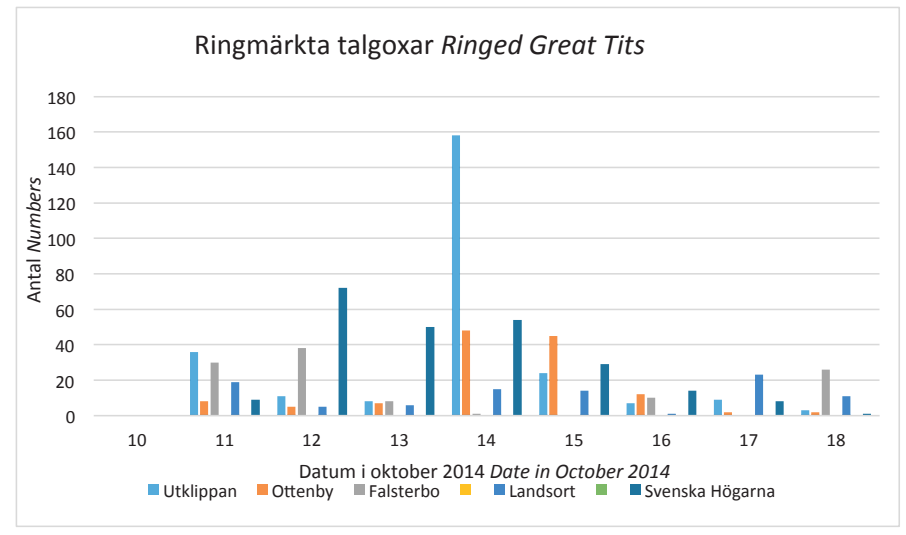


orientera sig och hålla sin sydvästliga flygriktning ha försvårats.

När solen gick ner över Östersjön strax före kl 18 svensk tid täckte regnområdet $\mathrm{i}$ stort sett hela södra och sydöstra Östersjön - söder om en linje från Ölands norra udde till Nida i Litauen. Om fåglarna då fortfarande befann sig över havet hade de inget annat val än att planlöst flyga omkring så länge de orkade i det dåliga vädret. Samtidigt vred vinden över till ost och sedan till kraftigt ökande nordost. Denna nordostvind torde ha drivit fåglarna ner mot sydväst.

Det är oklart när talgoxarna nådde Utklippan. Det måste antingen ha skett under eftermiddagen den 13:e då det regnade eller efter att regnet dragit bort (ca kl. 22) och under natten till den 14:e. Redan i gryningen den 14:e fanns de första talgoxarna på plats och under ungefär tre timmar fångades 158 talgoxar. Solen gick upp ca klockan 07:30 svensk sommartid. Om de lämnade den baltiska kusten på morgonen/förmiddagen den 13:e kan de ha varit i luften i 8 eller kanske till och med över 20 timmar.

\section{Viktminskning och flygtid}

Det är troligt att medelvikterna på de fyra olika lokalerna Utklippan, Torhamn, Flyet och Ottenby påverkas av andelen flyttande talgoxar (Tabell 1). Utklippan som är en utpräglad flyttfågellokal uppvisade de lägsta medelvikterna. Ottenby som också måste betecknas som i huvudsak en flyttfågellokal uppvisar inte fullt så låga medelvikter. Däremot har Flyet, som har en lokal population häckande talg-

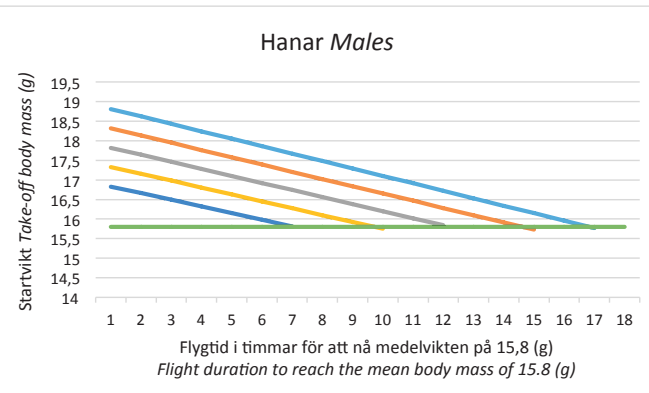

Figur 5. Beräknad flygtid för talgoxar (hanar) med olika startvikt för att nå den medelvikt som hanarna på Utklippan hade den 14 oktober 2014. Beräkningarna baseras på en viktminskning med $1 \%$ per flygtimme.

Estimated flight durations for Great Tits (males) in order to reach the mean body mass observed in males at Utklippan on October $14^{\text {th }} 2014$, calculated for different take-off body masses. Calculations are based on a body mass loss of $1 \%$ per flight hour. oxar, de högsta vikterna. Torhamn, som passeras av flyttande talgoxar men som också har en lokal population, hamnar mitt emellan. Samma mönster har noterats för kungsfåglar som fångats på samma lokaler (Larsson 2011).

Talgoxar, som under hösten ringmärkts vid två fågelstationer längs den polska kusten och som helt saknade fettreserver (synligt fett $=0$; Pettersson $\&$ Hasselquist 1985), hade en medelvikt (hanar och honor sammanslagna) på 16,62 respektive 16,67 g. De utgjorde 13,1 respektive $13,3 \%$ av totala antalet ringmärkta talgoxar (Scislowska \& Busse 2005). Eftersom dessa värden är för båda könen tillsammans bör siffrorna rimligtvis vara något för låga för hanarna och något för höga för honorna. Generellt väger hanarna i genomsnitt $1,1 \mathrm{~g}$ mer än honorna (Cramp 1993), vilket skulle ge 17,2 g för hanar och 16,1 g för honor utan fett. I Rybachy i det ryska Kaliningradområdet har den genomsnittliga vikten för talgoxar utan några fettreserver beräknats till 17,3 g för hanar och 16,3 $\mathrm{g}$ för honor (Dolnik \& Blyumental 1967).

Medelvikten för talgoxhanarna på Utklippan den 14 oktober var ca 1,5 g lägre jämfört med de hanar utan fett som fångats i Polen och vid Rybachy. För talgoxhonorna var motsvarande siffror för fettfri vikt i Polen/Rybachy väldigt lika, dvs 1,4-1,6 g lägre än på Utklippan den 14 oktober. Om man jämför med siffrorna från Polen/Rybachy saknade $90,4 \%$ av hanarna och $96,5 \%$ av honorna fettreserver på Utklippan 14 oktober 2014.

Enligt teoretiska beräkningar minskar sträckande fåglars kroppsvikt omkring $0,7 \%$ per flygtimme för de minsta tättingarna (Alerstam 1982). Som ett mått på viktminskning hos flyttande småfåglar

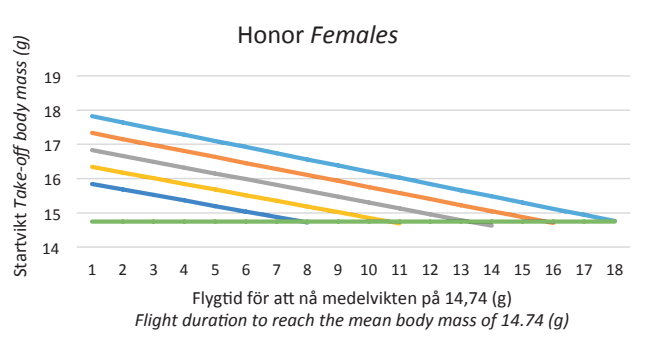

Figur 6. Beräknad flygtid för honor med olika startvikt för att nå den medelvikt som honorna på Utklippan uppvisade den 14 oktober 2014. Beräkningarna baseras på en viktminskning med $1 \%$ per flygtimme.

Estimated flight durations for Great Tits (females) in order to reach the mean body mass observed in females at Utklippan on October 14 ${ }^{\text {th }} 2014$, calculated for different take-off body masses. Calculations are based on a body mass loss of $1 \%$ per flight hour. 
såsom stenskvätta och trädgårdssångare har 1\% viktförlust per timmes flygning använts (Delingat et al. 2008, Barboutis et al. 2011). Notera dock att en studie av höststräcket längs Östersjöns kust $\mathrm{i}$ Baltikum visade på så höga värden som $2,2 \%$ viktförlust per flygtimme för bofink (Alerstam 1982). Bofinken är kortflyttare och det finns anledning att fråga sig om talgoxe, som ju också är kortflyttare, har samma dåliga bränsleekonomi som bofink.

Antag att en talgoxhane med en startvikt på 18,5 gram flyger i 8 timmar och har en viktminskning på $1 \%$ per timme. Då blir viktminskningen 1,48 gram efter 8 timmars flygning och 3,7 gram efter 20 timmars flygning. För en talgoxhona med en startvikt på 17,5 gram blir motsvarande siffror 1,4 respektive 3,5 gram.

För att nå den på Utklippan uppmätta medelvikten för hanarna - 15,8 $\mathrm{g}$ - behöver en hane med en startvikt på exempelvis 18,5 gram flyga i nästan 15 timmar. För en hona med startvikt 17,5 g tar det drygt 15 timmars flygning innan hon når den medelvikt på 14,74 g som talgoxhonorna på Utklippan uppvisade den 14 oktober 2014 (Figur 5 och 6).

Om man antar att talgoxarna den aktuella dagen/ natten tvingades flyga betydligt fler timmar än den teoretiska flygtiden på 8 timmar (med en hastighet av ca $40 \mathrm{~km} / \mathrm{h}$ ) för att tillryggalägga de 33 milen är det föga överraskande att en del av talgoxarna hade extremt låga vikter och att medelvikten stannade på 15,80 gram för hanarna och 14,74 gram för honorna. När de landade på Utklippan var de helt enkelt utmärglade och i desperat behov av föda. Detta förklarar också deras mycket aggressiva beteende. Det är också högst sannolikt att en del talgoxar som hade en mindre energireserv när de startade inte klarade överflygningen utan förolyckades innan de nådde den svenska kusten.

\section{Tack}

Till Hans Bengtsson på SMHI för hjälp med väderkartorna, Magnus Hellström på Ottenby fågelstation för biometriska uppgifter samt till Thord Fransson på Ringmärkningscentralen på Naturhistoriska riksmuseet för genomläsning av manus och statistiska beräkningar.

Väderdata (vindriktning och vindstyrka timme för timme) för den 13 och 14 oktober 2014 erhölls från: Estonian Meteorological and Hydrological Institute (Ristna), Latvian Environment, Geology and Meteorology Centre (Ventspils och Liepaja), Lithuanian Hydrometeorological Service (Klaipeda och Nida), SMHI (Utklippan, Ölands södra udde, Östergarn). Väderkartor för den 13 oktober över Östersjön kom från SMHI. Biometri för talgoxar ringmärkta vid Ottenby den 14 oktober 2014 erhölls från Ottenby fågelstation. Återfynd under perioden 14-17 oktober 2014 av talgoxar ringmärkta utomlands fick jag från Ringmärkningscentralen, Naturhistoriska Riksmuseet, Stockholm.

\section{Rererenser}

Alerstam, T. 1982. Fågelfyttning. Bokförlaget Signum i Lund AB, Uppsala.

Barboutis, C., Henshaw, I., Mylonas, M. \& Fransson, T. 2011. Seasonal differences in energy requirements of Garden Warblers Sylvia borin migrating across the Sahara desert. Ibis 153: 746-754.

Cramp, S. \& Perrins, C.M. (eds.) 1993. The Birds of the Western Palearctic. Vol. VII. Oxford University Press, Oxford.

Dolnik, V.R., Blyumental, T.I. 1967. Autumnal premigratory and migratory periods in the Chaffinch (Fringilla coelebs coelebs) and some other temperate-zone passerine birds. The Condor 69: 435-468.

Fransson, T. \& Hall-Karlsson, S. 2008. Svensk ringmärkningsatlas. Vol. 3. Stockholm.

Larsson, R. 2011. Höstflyttande kungsfåglars (Regulus regu$l u s)$ vikt på tre näraliggande rastplatser i sydöstra Sverige. Ornis Svecica 21: 20-28.

Lindholm, C.-G. 1978. Talgoxens sträck över Östersjön höstarna 1975 och 1976. Anser 3: 145-153.

Newton, I. 2008. The Migration Ecology of Birds. Academic Press, Elsevier, London.

Nowakowski J.K. 2001. Speed and synchronisation of autumn migration of the Great Tit (Parus major) along the eastern and the southern Baltic coast. Ring 23: 55-71.

Pettersson J., Hasselquist, D. 1985. Fat deposition and migrating capacity of robins Erithacus rubecula and goldcrests Regulus regulus at Ottenby, Sweden. Ringing \& Migration 6: 66-76.

Scislowska, M. \& Busse, P. 2005. Fat reserves and body mass in some passerines migrating in autumn through the southern Baltic coast. Ring 27: 3-59.

\section{Summary}

In the western Palearctic, the Great Tit is a sedentary species in the southern and central parts of its range, but invasive movements, sometimes with large numbers, occur irregularly in the northern parts. Many of the Great Tits nesting in the north-western parts of Russia migrate in autumn, mainly to the Baltic Sea region but often even longer (Cramp 1993). Normally, Great Tits are day-time migrants (Newton 2008). Based on ringing data from Poland, $70 \%$ of the retrapped Great Tits had a migration speed of 18-44 km per day (Nowakowski 2001). However, the latter estimate is determined, besides flight speed, also by the time spent on stop over and feeding. In terms of flight 
speed alone, the top flight speed for a Great Tit in strong tailwind may reach $84 \mathrm{~km}$ per hour (Alerstam 1982). There was no relationship between migration intensity and movement speed of the great tit (Nowakowski 2001).

\section{The weather situation}

During daylight on October 13th the winds were weak, $<5 \mathrm{~m} / \mathrm{s}$ and blowing from west at the eastern coast of the Baltic Sea. In the Gulf of Gdansk there were very weak winds and an area of fog and mist in the morning hours. At the same time an area of rain approached from SW the southern and southeastern parts of the Baltic Sea (Figure 1 and 2). In the afternoon the wind turned to E-NE on the central parts of the Baltic Sea and during the night the wind speed increased to $12-14 \mathrm{~m} / \mathrm{s}$.

\section{The event}

On 13-14 October 2014, in prevailing easterly to north-easterly winds, a massive influx of Great Tits occurred at Utklippan, an island $15 \mathrm{~km}$ to the south-southwest of Torhamn in the province of Blekinge off the south-eastern coast of Sweden. During these days Great Tits were predominant in the catch at Utklippan Bird Observatory and all of them were in poor condition and weighed far below what is normal for this time of the year. Several of the emaciated birds died on the island while others were very aggressive and attacked and killed other birds apparently in the struggle to find food.

On Utklippan very few Great Tits $(n=8)$ were trapped on October 13th before the rain reached the island at $2 \mathrm{pm}$ local time (CEST). At dawn and the early morning hours on October 14th 158 Great Tits were trapped, ringed and weighed. The mist nets were closed at $10.30 \mathrm{am}$ local time due to the aggressiveness of the Great Tits.

\section{Body mass patterns}

At Utklippan October 14th 2014, most of the Great Tits were lean and had very low body mass. On this day, the captured males had a mean body mass of $15.80 \mathrm{~g}(\mathrm{n}=73)$. This was considerably lower than the mean body mass for males during October 2007-2013 at Utklippan (16.99 g; U-test, $\mathrm{z}=7.82$, $\mathrm{p}<0.001)$, as well as at Torhamns udde $(17.53$ g) and Flyet (18.54 g; Figure 3 and Table 1). The mean body mass of the females at Utklippan October 14th was $14.74 \mathrm{~g}(\mathrm{n}=85)$. This was also much lower than during October 2007-2013 at Utklippan
(15.95 g; U-test, $\mathrm{z}=3.19, \mathrm{p}<0.001)$ as well as at Torhamns udde (16.43 g) and Flyet, an upland site $2 \mathrm{~km}$ from the coast and $10 \mathrm{~km}$ north-northeast of Torhamn (17.33 g; Figure 3 and Table 1).

At Ottenby Bird Observatory on the large island of Öland in the southwest Baltic Sea, 47 Great Tits were trapped October 14th. The mean body mass of both the males and the females were higher than those of the Great Tits at Utklippan the same day. The mean body mass of the males was 16.51 (n $=26$ ) and this was significantly different from the mean body mass of Great Tit males at Utklippan (U-test, $\mathrm{z}=3.19, \mathrm{p}=0.001$ ). For female Great Tits caught October 14th 2014, the mean body mass at Ottenby was $15.03 \mathrm{~g}(\mathrm{n}=13)$ and it did not differ significantly from mean body mass of females at Utklippan (U-test, $\mathrm{z}=1.46, \mathrm{p}=0.15$ ).

Utklippan and Ottenby should mainly have been catching migrating Great Tits, because neither of these sites have any local breeding population of Great Tits. In contrast, Torhamn and Flyet are located at the Swedish mainland along the southwestern Baltic Sea coast, and both these sites have large breeding populations of Great Tits nearby. This may explain why the mean body mass of Great Tit males and females trapped at Torhamn and Flyet during October 2007-2013 were clearly higher than the mean body mass of great Tits caught at Utklippan over the same period (Table 1 and 2; Kruskal Wallis test, males, $\mathrm{H}$ $=315.4, \mathrm{df}=2, \mathrm{p}<0.001$; females $\mathrm{H}=348.0, \mathrm{df}$ $=2, \mathrm{p}<0.001)$.

\section{Aggressive behavior}

On the ground at Utklippan Great Tits were found dead. Several Great Tits were so exhausted that they died in the mist nets, even if the nets were under constant supervision. Great Tits were also seen eating on dead conspecifics and they also attacked other species trapped in the nets. They started with pecking at the head of the victim to eat the brain. They also ate the breast muscle.

\section{Previous observations of influx of aggressive Great Tits}

Occasional influxes of Great Tits are not frequent. I am only aware of two such events, one in October 2002 and another in October 2011. The mean body mass of males was $17.01 \mathrm{~g}(13.9-20.5 \mathrm{~g})$ in 2002 and $17.28 \mathrm{~g}(14.2-20.5 \mathrm{~g})$ in 2011. The mean body mass of females was $15.78 \mathrm{~g}(13.1-18.5 \mathrm{~g})$ in 2002 and $16.25 \mathrm{~g}(13.4-19.0 \mathrm{~g})$ in 2011 . These 
numbers are clearly higher than those observed on October 14th 2014 at Utklippan. One reason for this can be that the Great Tits in 2002 and 2011 had rather strong tailwinds when flying across the Baltic Sea, whereas this was not the case 13-14 October 2014.

\section{From where did the lean Great Tits come?}

The influx of Great Tits to Utklippan is likely to be part of an influx of passerine birds from the eastern shore of the Baltic Sea. At several bird observatories along the East coast of Sweden, large numbers of Great Tits were trapped and ringed 14-18 October (Figure 4). Several recoveries of Great Tits ringed in Finland (2), Lithuania (1), Russia (1) and Poland (2) were made at Swedish bird observatories during this period. One Russian bird ringed on October 9th 2014 was trapped October 16th at Ottenby. Another Great Tit recaptured at Ottenby October 14th 2014 was ringed in October 2013 in Poland. A bird ringed in Poland October 10th 2014 was recaptured at Falsterbo, southwestern Scania 8 days later. The two great Tits from Finland were recovered at Svenska högarna 3 and 6 days after they were ringed at Hanko in the Gulf of Finland.

The Great Tits that were trapped at Utklippan early in the morning October 14th ought to have departed from the eastern coast of the Baltic Sea north of Lithuania sometime in the morning October 13th despite the weak W-SW headwinds. It is not probable that they departed from Poland, the Kaliningrad area or the southern part of Lithuania, because this area was covered with mist and fog until noon (Figure 1).

At departure, the Great Tits should have faced a weak headwind that slowed down their flight speed. Well out over the sea, they should have encountered the cloud screen that preceded the area of rain that was approaching from southwest. As a consequence, the ability of the birds to orient and to stay on their track towards SW may have been severely reduced.

At sunset shortly before 18 CEST the area of rain covered nearly all parts of the S and SE of the Baltic Sea (Figure 2). If the birds still were flying over the sea at this point, they may only have had the option to continue flying as long as they could in the bad weather to find land where they could stop over. At the same time the wind turned to E and later to NE and increased severely in speed. This NE wind might have driven the birds that were flying over the Baltic Sea towards SW.

The Great Tits must have reached Utklippan in the rain in the afternoon October 13th or during the following night. They may then have been flying non-stop for at least 8 hours, and maybe even for as long as 20 hours, to reach Utklippan.

\section{Travelling distances and body mass loss}

At two Polish bird observatories the lean body mass of Great Tits was calculated to be 16.62 and $16.67 \mathrm{~g}$ (males and females combined) and the birds without any fat reserves made up ca. $13 \%$ of all ringed Great Tits (Scislowska \& Busse 2005). In general, the males are $1.1 \mathrm{~g}$ heavier than the females (Cramp 1993), and if I use this value to recalculate the figures above this means that the lean body mass for Polish Great Tit males is $17.2 \mathrm{~g}$ and for females $16.1 \mathrm{~g}$. At Rybachy (Kaliningrad), the average lean body mass of Great Tits was $17.3 \mathrm{~g}$ for males and $16.3 \mathrm{~g}$ for females (Dolnik \& Blyumental 1967).

The average body mass of the males trapped at Utklippan on October 14th was $1.5 \mathrm{~g}$ lower, and for the females nearly 1.4-1.6 g lower, than the Great Tits without any fat reserves in the above-mentioned studies from Poland and Rybachy. $90.4 \%$ of the males and $96.5 \%$ of the females at Utklippan had no fat reserves at all, based on the mean lean body mass values from Poland and Rybachy.

According to theoretical calculations the body mass of migrating small passerines decreases with about $0.7 \%$ per hour of flight (Alerstam 1982). In studies of migrating small passerines, such as the Northern Wheatear Oenanthe oenanthe and the Garden Warbler Sylvia borin, a body mass loss of $1 \%$ per hour flight has been used (Delingat et al. 2008, Barboutis et al. 2011).

If we assume that a male Great Tit have a starting body mass of $18.5 \mathrm{~g}$ and a body mass loss of 1 $\%$ per hour of flight, then his body mass loss will be $1.48 \mathrm{~g}$ after 8 hours and $3.7 \mathrm{~g}$ after 20 hours of flight. Accordingly, a female with a starting body mass of $17.5 \mathrm{~g}$ will lose $1.4 \mathrm{~g}$ after 8 hours and 3.5 $\mathrm{g}$ after 20 hours flight. Based on these calculations, a male with an assumed body mass at take-off of $18.5 \mathrm{~g}$ will have needed to fly non-stop for nearly 15 hours to reach the mean body mass recorded at Utklippan (15.8 g). Similarly, for a female with a body mass of $17.5 \mathrm{~g}$ at take-off, she would have needed to fly non-stop for more than 15 hours to reach the mean body mass recorded at Utklippan (14.75 g; Figure 5 and 6).

If we believe that the Great Tits reaching Utklippan on October 13th and 14th were forced to fly for much longer than the expected 8 hours (calculation 
based on a velocity of approximately $40 \mathrm{~km} / \mathrm{h}$; Alerstam 1982) to cover the $330 \mathrm{~km}$ over the Baltic Sea, it is not surprising that the body mass of many Great Tits were extremely low. When these Great Tits reached Utklippan they should have been ema- ciated and in desperate need of food, which may explain their unusual aggressiveness. Probably some Great Tits with low take-off fat reserves did not even make the crossing of the Baltic Sea to reach the Swedish coast. 